\title{
Cycling of dense core vesicles involved in somatic exocytosis of serotonin by leech neurons
}

\author{
Citlali Trueta ${ }^{1}$, Damien P. Kuffler ${ }^{2}$ and Francisco F. De-Miguel ${ }^{3}$ * \\ "Instituto Nacional de Psiquiatría "Ramón de la Fuente Muñiz," México D. F., México \\ ${ }^{2}$ Institute of Neurobiology, University of Puerto Rico, San Juan, Puerto Rico \\ ${ }^{3}$ Instituto de Fisiología Celular-Neurociencias, Universidad Nacional Autónoma de México, México D. F., México
}

\section{Edited by:}

Kjell Fuxe, Karolinska Institutet,

Sweden

Reviewed by:

Kjell Fuxe, Karolinska Institutet,

Sweden

Diego Guidolin, University of Padova, Italy

*Correspondence:

Francisco F. De-Miguel, Instituto de Fisiología Celular-Neurociencias,

Universidad Nacional Autónoma de México, Circuito Exterior, Ciudad

Universitaria, 04510 México D. F.,

Mexico.

e-mail: ffernand@ifc.unam.mx
We studied the cycling of dense core vesicles producing somatic exocytosis of serotonin. Our experiments were made using electron microscopy and vesicle staining with fluorescent dye FM1-43 in Retzius neurons of the leech, which secrete serotonin from clusters of dense core vesicles in a frequency-dependent manner. Electron micrographs of neurons at rest or after $1 \mathrm{~Hz}$ stimulation showed two pools of dense core vesicles. A perinuclear pool near Golgi apparatuses, from which vesicles apparently form, and a peripheral pool with vesicle clusters at a distance from the plasma membrane. By contrast, after $20 \mathrm{~Hz}$ electrical stimulation $47 \%$ of the vesicle clusters were apposed to the plasma membrane, with some omega exocytosis structures. Dense core and small clear vesicles apparently originating from endocytosis were incorporated in multivesicular bodies. In another series of experiments, neurons were stimulated at $20 \mathrm{~Hz}$ while bathed in a solution containing peroxidase. Electron micrographs of these neurons contained gold particles coupled to anti-peroxidase antibodies in dense core vesicles and multivesicular bodies located near the plasma membrane. Cultured neurons depolarized with high potassium in the presence of FM1-43 displayed superficial fluorescent spots, each reflecting a vesicle cluster. A partial bleaching of the spots followed by another depolarization in the presence of FM1-43 produced restaining of some spots, other spots disappeared, some remained without restaining and new spots were formed. Several hours after electrical stimulation the FM1-43 spots accumulated at the center of the somata. This correlated with electron micrographs of multivesicular bodies releasing their contents near Golgi apparatuses. Our results suggest that dense core vesicle cycling related to somatic serotonin release involves two steps: the production of clear vesicles and multivesicular bodies after exocytosis, and the formation of new dense core vesicles in the perinuclear region.

Keywords: serotonin, somatic exocytosis, extrasynaptic exocytosis, dense core vesicle, vesicle cycle, volume transmission, endocytosis, leech

\section{INTRODUCTION}

Depolarization of the soma of serotonergic neurons induces serotonin secretion via the mobilization of serotonin-containing vesicles from central neuronal regions to the plasma membrane (Trueta et al., 2003; De-Miguel and Trueta, 2005; Kaushalya et al., 2008; Colgan et al., 2009). Serotonin in the soma of leech Retzius neurons, a classic preparation in the study of serotonin release (for Review, see Nicholls and Kuffler, 1990; Fernández-de-Miguel and Drapeau, 1995), is packed in clusters of dense core vesicles that, in response to brief trains of electrical impulses at high frequency are transported toward the plasma membrane (Trueta et al., 2004), with which they fuse during the following minutes. Vertebrate serotonergic neurons, including those in mammalians also pack serotonin into extrasynaptic clear and dense core vesicles (Chazal and Ralston, 1987; Descarries and Mechawar, 2000), and something similar happens in neurons containing dopamine, adrenaline, and peptides (see review by Trueta and De-Miguel in this issue). This mode of exocytosis may be responsible for volume transmission within the nervous system (for review, see Fuxe and Agnati, 1991; Agnati et al., 2006).
Although the mechanism of somatic secretion described for Retzius neurons seems to be part of a more general principle of somatic and extrasynaptic exocytosis in vertebrates and invertebrates (see review by Trueta and De-Miguel in this issue), the cycling of serotonin-containing vesicles has still not been elucidated. While clear vesicles at synapses may be reloaded and reused rapidly, within seconds after their fusion with the plasma membrane (Betz and Bewick, 1992, 1993; Betz et al., 1992; Cochilla et al., 1999; Richards et al., 2000), dense core vesicles undergo a longer cycle before their reuse (Herzog and Farquhar, 1977; Herzog and Miller, 1979; Patzak et al., 1987; Ceridono et al., 2011). Multiple studies have discussed the molecular mechanisms that contribute to the vesicle cycle in different types of cells (for review, see Piper and Katzmann, 2007; Park et al., 2009; Von Bartheld and Altick, 2011). However, since serotonin is reloaded into clear vesicles by vesicular transporters (Iversen, 1971), one question is whether somatic dense core vesicles are rapidly reused after exocytosis or do they enter the long cycle typically characterized for dense core vesicles. The focus in this study was to provide a descriptive overview of the basic steps of the vesicle cycling that leads to the formation, 
transport, fusion, retrieval, and recycling of serotonin-containing dense core vesicles.

We addressed this question by taking advantage of the large size of serotonergic leech Retzius neurons, which have the largest somata in the ganglia and are the major serotonin-producing neurons in the leech nervous system. Because of their large (60$80 \mu \mathrm{m}$ ) soma diameters and the possibility of studying them within intact ganglia as well as in isolation in tissue culture, they are an excellent preparation on which to study the events underlying somatic exocytosis of serotonin.

Retzius neurons in the intact animal and in culture synthesize and pack serotonin in clear small $(40 \mathrm{~nm})$ synaptic vesicles and in large $(100 \mathrm{~nm})$ dense core vesicles that are distributed in the soma, extended processes, and perisynaptic regions (Henderson, 1983; Kuffler et al., 1987; Bruns and Jahn, 1995). The soma contains "astronomic" (Coggeshall, 1972) numbers of serotonincontaining dense core vesicles that at rest are clustered away from the plasma membrane (Trueta et al., 2004). Previous studies using staining of vesicles that undergo exocytosis followed by endocytosis in the presence of the fluorescent dye FM1-43 and electron microscopy have shown that electrical stimulation of Retzius neurons with trains of pulses at 10 or $20 \mathrm{~Hz}$, but not $1 \mathrm{~Hz}$, evoke calcium-dependent vesicle cluster mobilization followed by the fusion of vesicles with specific areas of the plasma membrane (Trueta et al., 2003, 2004).

To study the cycling of dense core vesicles from their perinuclear location at rest and for several hours after they had undergone exo- and endocytosis we used electron microscopy of neurons in the ganglion and the staining of vesicles by fluorescent FM1-43 dye as they fuse with the plasma membrane of cultured neurons. Electron microscopy was used to follow the sites of vesicle formation, their mobilization and fusion in response to depolarization, and their process of recycling after endocytosis.

\section{MATERIALS AND METHODS ISOLATION AND CULTURE OF NEURONS}

Retzius neurons were isolated from the central nervous system of adult leeches Hirudo sp. The procedure has been described elsewhere (Dietzel et al., 1986). In brief, leeches were anesthetized by immersion in $8 \%$ ethanol. Nerve cords were dissected out and ganglion capsules opened to expose the cell somata. Ganglia were kept in Leibovitz L-15 culture medium, supplemented with $6 \mathrm{mg} \mathrm{ml}^{-1}$ glucose, $0.1 \mathrm{mg} \mathrm{ml}^{-1}$ gentamicin and $2 \%$ heatinactivated fetal calf serum, and they were incubated for $1 \mathrm{~h}$ in $2 \mathrm{mg} \mathrm{ml}^{-1}$ collagenase/dispase solution (Boehringer-Mannheim, Mannheim, Germany). After enzyme treatment, Retzius neurons were sucked out one by one, rinsed several times in L-15, and then plated on glass bottomed culture dishes precoated with concanavalin-A. Experiments were performed after the neurons were $1-8$ days in culture.

\section{STIMULATION OF EXOCYTOSIS}

Single trains of electrical stimulation were used for FM1-43 staining experiments, while series of 10 trains were used for studies to be evaluated in the electron microscope. One stimulation protocol involved the application of trains of 10 action potentials produced by intracellular injection of $20-\mathrm{ms}$ current pulses at 1 , or $20 \mathrm{~Hz}$, using borosilicate microelectrodes with resistances of $18-25 \mathrm{M} \Omega$ when filled with $3 \mathrm{M} \mathrm{KCl}$. Electrical recordings were acquired by an analog-to-digital board Digidata 1200 (Axon Instruments) at a sampling frequency of $20 \mathrm{kHz}$, using pCLAMP8 software (Axon Instruments) and the data was stored in a computer. Before withdrawing the microelectrode, the dye was washed out of the bathing solution for $2 \mathrm{~min}$ with physiological saline solution ( $\mathrm{mM}: \mathrm{NaCl}$ 120; $\mathrm{KCl} 4 ; \mathrm{CaCl}_{2} 2$; Tris-maleate $10 ; N$-methyl-D-glucamine 66) in the absence of $\mathrm{Ca}^{2+}$, which was replaced by magnesium $(2 \mathrm{mM})$ to reduce vesicle fusion induced by these manipulations. To test the $\mathrm{Ca}^{2+}$ dependence of serotonin secretion, neurons were maintained in an external bathing solution containing $2 \mathrm{mM} \mathrm{Mg}^{2+}$ instead of $\mathrm{Ca}^{2+}$. N-methyl-D-glucamine was added to the bathing solution to adjust it's osmolarity to $330 \mathrm{mosmol}^{-1}$. After the electrode was withdrawn, the cells were washed for $8 \mathrm{~min}$ with normal Ringer's solution.

\section{ELECTRON MICROSCOPY}

Neurons in the ganglion were stimulated with 10 trains of 10 impulses each at 1 or $20 \mathrm{~Hz}$ frequencies separated by $1 \mathrm{~min}$ intervals to evoke exocytosis from the external and internal vesicle pools. Some neurons were also stimulated using external solutions containing magnesium instead of calcium. Ganglia were then perfused with $0.08 \mathrm{M}$ cacodylate buffer ( $\mathrm{pH} 7.4$; Sigma, St. Louis, MO, USA), and fixed for $60 \mathrm{~min}$ with $0.6 \%$ glutaraldehyde and $0.4 \%$ paraformaldehyde, followed by postfixation for $60 \mathrm{~min}$ in $1.0 \%$ osmium tetroxide (Kuffler et al., 1987). Thin 70-100 nM sections usually containing the nucleus were observed in a JEOL 1010 electron microscope (JEOL USA Inc., Peabody, MA, USA). For illustration, electron micrographs were digitized at $1200 \mathrm{dpi}$.

\section{PEROXIDASE IMMUNODETECTION IN ELECTRON MICROSCOPY}

Ganglia were opened with forceps to expose the Retzius neurons. After a $1-\mathrm{h}$ incubation in $10 \mathrm{mg} / \mathrm{ml}$ peroxidase (Sigma) in the extracellular fluid, one of the neurons in the ganglion was electrically stimulated. Some ganglia were fixed during neuronal stimulation, while others were fixed $10 \mathrm{~min}$ after electrical stimulation had stopped. After fixation, ganglia were washed in PBS and fixed as described above. Ultrathin sections were obtained with a Reichert-Jung ultramicrotome calibrated to produce $50-70 \mathrm{~nm}$ thick sections, according to their interference silver color. Once mounted on nickel grids, sections were incubated with Tris-BSA supplemented with $1 \%$ Triton for $15 \mathrm{~min}$, followed by the addition of $10 \%$ goat serum for $15 \mathrm{~min}$. A primary anti-peroxidase rabbit antibody (Sigma) was added at a dilution of 1:50 for a final concentration of $0.2 \mathrm{mg} / \mathrm{ml}$. After a 12-h incubation at room temperature, sections were washed with Tris-BSA medium and then incubated for $1 \mathrm{~h}$ with a secondary goat polyclonal anti-rabbit antibody coupled to $10 \mathrm{~nm}$ gold spheres (Sigma) at a 1:50 dilution. Control sections were incubated with goat serum. The sections were washed and contrast stained using lead citrate and uranyl acetate.

\section{FM1-43 VESICLE STAINING}

Exocytosis from living neurons was analyzed as described by Trueta et al. (2003), following the incorporation into vesicles of the fluorescent dye FM1-43 (Molecular Probes; Betz and Bewick, 1992; 
Betz et al., 1992). FM1-43 (2 $\mu \mathrm{m})$ was added to the bathing solution after the neurons had been impaled with a microelectrode and hyperpolarized to $-60 \mathrm{mV}$ to prevent spontaneous electrical activity. The rest of the stimulation protocol was described above.

In FM1-43 destaining and restaining experiments, sequential images of fluorescently labeled spots within the neuron soma were captured while the solution bathing the cultured neurons was changed to one containing $40 \mathrm{mM} \mathrm{K}^{+}$. For this, FM1-43 was added to the tissue culture plates containing $1 \mathrm{ml}$ of normal Ringer's solution, followed by the addition of $1 \mathrm{ml}$ of modified Ringer's solution in which $76 \mathrm{mM} \mathrm{NaCl}$ was replaced by $\mathrm{KCl}$ by equimolar substitution, to reach a final concentration of $40 \mathrm{mM}$ potassium. After $5 \mathrm{~min}$, the dye was washed out. Some neurons were stimulated in $\mathrm{Mg}^{2+}$ solution. Other neurons were incubated with FM1-43 in normal (1.8 mM) $\mathrm{Ca}^{2+}$ Ringer without stimulation, or with $40 \mathrm{mM} \mathrm{K}^{+}$solution in the absence of FM1-43. The recording chamber was perfused by gravity feed and complete solution changes took $30 \mathrm{~s}$.

\section{FLUORESCENCE IMAGING}

Individual neurons were viewed with a Nikon Eclipse TE 200 microscope through a Nikon $\times 100$ oil immersion objective (NA 1.25). Neutral density filters reduced the illumination intensity by $90 \%$ to reduce photobleaching of the dye and neuronal damage. Fluorescence imaging of FM1-43-stained cells was performed with band-pass filters for excitation (peak $480 \mathrm{nM}$ ) and emission (peak $535 \mathrm{nM}$ ). Images were acquired by a CCD camera (Hamamatsu Photonics, Japan) coupled to an Argus 10 integrator (Hamamatsu Photonics) programmed to integrate from 128 to 256 images. In destaining/restaining experiments, the number of fluorescent FM1-43 labeled spots, which correspond to clusters of vesicles that underwent exo/endocytosis (Trueta et al., 2003), were manually counted using stereological criteria (Coggeshall and Lekan, 1996).

\section{TIME LAPSE IMAGING OF FM1-43 FLUORESCENCE}

Neurons were stimulated with $20 \mathrm{~Hz}$ trains in the presence of FM143 , and $10 \mathrm{~min}$ after stimulation the extracellular dye was washed out as described and were imaged at different $Z$ planes as described every $12 \mathrm{~h}$ over $48 \mathrm{~h}$.

\section{RESULTS}

\section{GENERAL ULTRASTRUCTURE OF THE SOMA OF RETZIUS NEURONS}

Ultra thin sections from five somata that had been stimulated in the ganglion with trains at $1 \mathrm{~Hz}$, a frequency that does not evoke somatic exocytosis, contained dense core vesicle clusters distributed in two main areas (Figures 1 and 2). A perinuclear region contained vesicles together with Golgi apparatuses, mitochondria, and endoplasmic reticulum. Small clear vesicles were located next to Golgi apparatuses and some small vesicles displayed dense cores. At larger distances from Golgi, vesicles were large and had dense cores. Multivesicular bodies were mainly located adjacent to the perinuclear vesicle clusters and were characterized by their bag shapes and membrane multilayers filled with dense core and clear vesicles (Figure 1, see also below).

A more peripheral pool of dense core vesicles consisted of clusters located between the perinuclear vesicle layer and the plasma membrane (Figures 1 and 2). Vesicles in these clusters appeared at different distances from the plasma membrane and were associated with microtubule fibers characterized by individual diameters of $20-25 \mathrm{~nm}$ (Figure 1C). These vesicle clusters were also associated with mitochondria and endoplasmic reticulum. The space between the vesicle clusters and the plasma membrane had only sporadic dense core vesicles and was occupied by smooth endoplasmic reticulum. In some sections microtubule bundles associated with the vesicle clusters appeared anchored to hemidesmosomes arriving at the plasma membrane in places where it usually formed invaginations (Figure 1C). The whole extracellular periphery of Retzius neurons was surrounded by layers of processes of giant segmental glia (Coggeshall and Fawcett, 1964), which were assembled in fingers that filled the invaginations of the Retzius cells. Similar structures were found in non-stimulated neurons, neurons stimulated at $1 \mathrm{~Hz}$ (Figures $\mathbf{1 A}, \mathbf{C}$ ), or neurons stimulated at $20 \mathrm{~Hz}$ in the presence of magnesium substituting for calcium in the external solution (Figure 2A).

In six neurons fixed after $20 \mathrm{~Hz}$ stimulation, $47 \%$ of the vesicle clusters were apposed to the plasma membrane (Figure 1E), vs. the $9 \%$ of vesicle clusters in neurons stimulated at $1 \mathrm{~Hz}$ (Figure 1E). The perinuclear clusters remained in the same region. In the plasma membrane, some vesicles had omega-like appearances indicative of vesicles that had fused with the plasma membrane in response to the electrical stimulation (Figure 4). Multivesicular bodies were commonly located within peripheral vesicle clusters and were surrounded by radial arrays of dense core vesicles (Figure 1D), suggesting that multivesicular bodies had been formed by endocytosis following exocytosis.

\section{FLUORESCENT FM1-43 LABELING OF VESICLES UPON EXO- AND ENDOCYTOSIS}

Electrical stimulation of cultured Retzius neurons with a $20-\mathrm{Hz}$ train in the presence of FM1-43 in the external fluid produces a spotted fluorescent pattern, in which each fluorescent spot is due to exocytosis followed by endocytosis from a dense core vesicle cluster (Trueta et al., 2003). The fact that the FM1-43 fluorescence in Retzius neurons is diminished as a result of prolonged depolarization by high extracellular potassium solution has suggested that vesicles in the clusters may undergo a second round of vesicle fusion (Trueta et al., 2003). However, a plausible alternative is that the fluorescence is reduced as vesicles become transported back to more central parts of the neuronal soma. In addition, the finding that electrical stimulation at increasing frequencies increases the number of fluorescent spots suggests that new clusters of vesicles are transported to and fuse at different release sites (Trueta et al., 2003). These possibilities were explored in four neurons in which the fluorescent FM1-43 spots formed upon high potassium depolarization were partially bleached by continuous light exposure followed by additional high potassium depolarization in the presence of fresh FM1-43. When counting the number of fluorescent spots in the equatorial plane of the soma in the same focal plane of the neurons before and after the second depolarization, we found four complementary effects (Figure 3): (1) 41\% of the spots became restained, suggesting either, the arrival of a second vesicle cluster, maybe from the perinuclear region of the cell, or a second round of fusion of the same vesicles. However the presence 


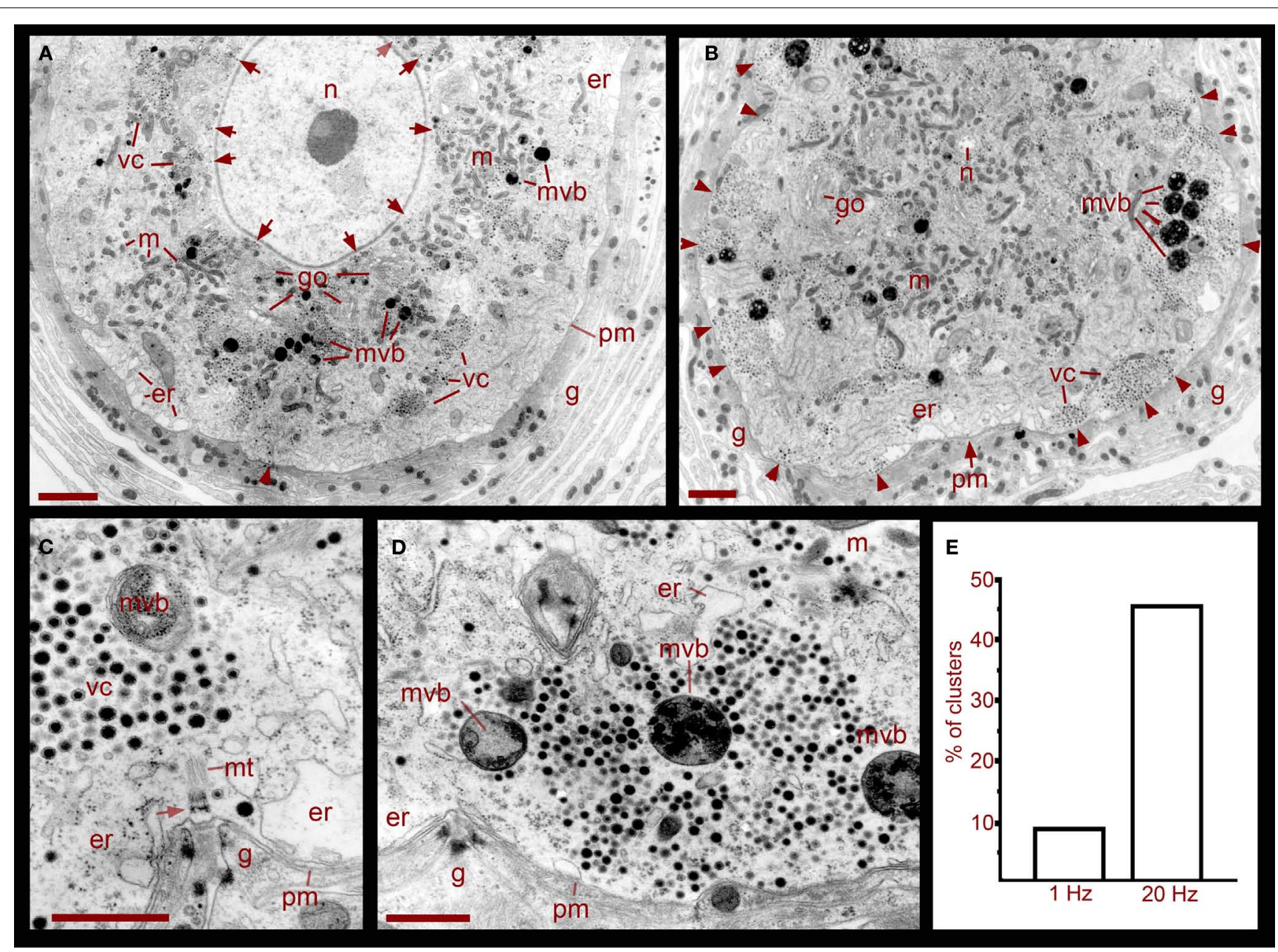

FIGURE 1 | Ultrastructure of the soma of Retzius neurons in relation to somatic exocytosis. (A) Low magnification electron micrograph of a neuron that had been stimulated at $1 \mathrm{~Hz}$, a frequency that fails to evoke somatic serotonin exocytosis. Vesicle clusters located around the nucleus (n) are indicated with arrows. Clusters in the region midway between the nucleus and the plasma membrane are indicated as vc. Vesicle clusters and multivesicular bodies (mvb) are absent in the peripheral cytoplasmic area next to the plasma membrane. Some multivesicular bodies are interspersed within the vesicle clusters. Golgi apparatuses (go) are also more concentrated around the nucleus. Mitochondria $(\mathrm{m})$ are near vesicles and endoplasmic reticulum (er) are located near the plasma membrane (pm). The arrowhead points to microtubule bundle arriving at the plasma membrane. The neuron is surrounded by layers of the giant glial cell (g). Scale $=2 \mu \mathrm{m}$. (B) Electron micrograph of a neuron stimulated at $20 \mathrm{~Hz}$. A majority of the vesicle clusters are apposed to the plasma membrane (arrowheads). Multivesicular bodies lie within peripheral vesicle clusters and mitochondria can be seen around them (small arrows). Note that even after stimulation, vesicle clusters remained near the nucleus (n). Scale $=2 \mu \mathrm{m}$. (C) Magnified electron micrograph of a peripherally located vesicle cluster in a neuron that had been stimulated at $1 \mathrm{~Hz}$. Vesicle clusters are located away from the plasma membrane and the intermediate space contains endoplasmic reticulum. A bundle of microtubules (mt) extends from the vesicle cluster vesicles to a hemi-desmosome (arrow) apposed to an invagination of the plasma membrane (arrowheads). Glial cell fingers characteristic of these structures penetrate into the somatic invagination. Scale $=1 \mu \mathrm{m}$. (D) Magnified electron micrograph of a neuron stimulated at $20 \mathrm{~Hz}$. A vesicle cluster is apposed to the plasma membrane near an invagination (arrowheads). Vesicle rows formed radial arrays with multivesicular bodies. Scale $=1 \mu \mathrm{m}$. (E) Graph of the percentage of vesicle clusters apposed to the plasma membrane in neurons stimulated at frequencies of 1 vs. $20 \mathrm{~Hz}$. Individual clusters were identified and counted from digital images low magnification and scanned at high resolution such as those in (A) and (B). As seen in (C,D), this vesicle cluster counting could be done easily since the clusters are self-delimited and they only contain vesicles and multivesicular bodies. of two pools of vesicle clusters and their correlation with the double sigmoidal kinetics of the FM1-43 fluorescence (P. Noguez, C. Bustos, and F. F. De-Miguel, in preparation) support the idea of the arrival and fusion of a second vesicle cluster over the reuse of the same dense core vesicles. The following three evidences provide further support to this possibility. (2) 19\% of the spots that were bleached were not restained in response to the second depolarization, although they stayed at the same position. (3) $22 \%$ percent of the prestained spots disappeared, suggesting their intracellular transport out of the focal plane. (4) New spots were formed in places that were previously not stained, accounting for $18 \%$ of the total final spots. This was consistent with the recruitment of new vesicle clusters that were fused at new release sites, as it happens with increases in the frequency of stimulation (see Figure 4 in Trueta et al., 2003) and suggest the slow vesicle recycling and the transport of fused vesicles back to internal cell sites. 


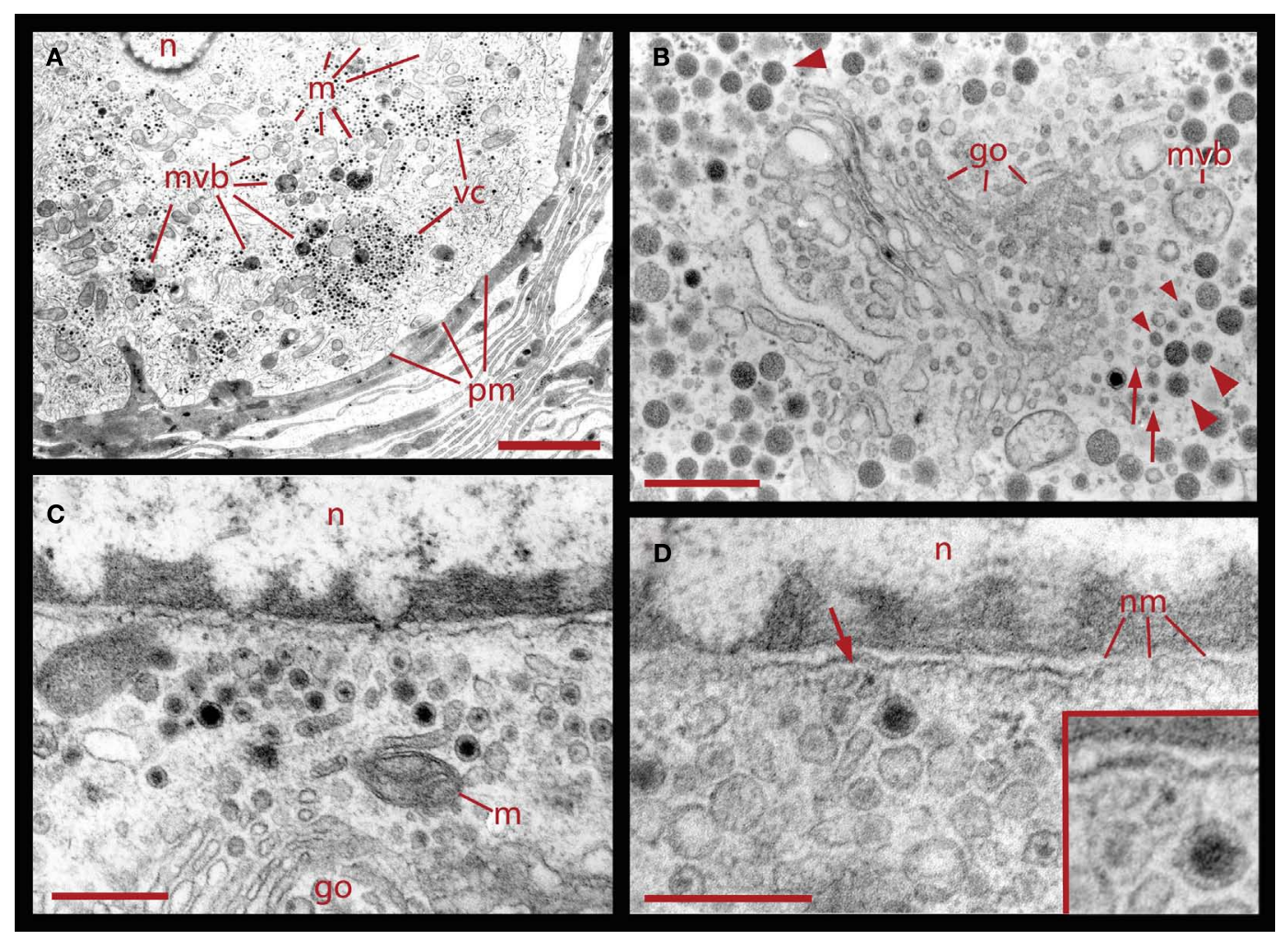

FIGURE 2 | Ultrastructural relationships of perinuclear vesicles with the nucleus (n), Golgi (go), and vesicle clusters (vc). (A) LoW

magnification image of the soma of a neuron that had been stimulated with $20 \mathrm{~Hz}$ trains in a bathing solution in which magnesium was

substituted for calcium. Under these conditions, electrical activity fails to evoke somatic exocytosis and vesicle mobilization, and as can be seen, the areas immediate to the plasma membrane $(\mathrm{pm})$ are devoid of vesicle clusters. Scale $=2.0 \mu \mathrm{m}$. (B) Golgi apparatus in the perinuclear area surrounded by clear and dense core vesicles. On the right, there are rows of small vesicles (arrows), some of which are clear while others have electron dense material (small arrowheads). More distantly there are small vesicles with dense cores continued by vesicles with large dense cores (large arrowheads). A fragment of a multivesicular body (mvb) can also be seen. On the top left electron dense vesicles align with the layers of the Golgi apparatus (large arrowhead). (C) Higher magnification image of vesicles adjacent to the nucleus, in the vicinity of mitochondria $(\mathrm{m})$ and Golgi. (D) Clear and dense core vesicles are adjacent to the nuclear membrane $(\mathrm{nm})$. A filament projecting from the vesicle toward the nuclear membrane is marked by the arrow and amplified in the inset. Scale bars for $($ B-D) $=500 \mathrm{~nm}$.

\section{ENDOCYTOSIS FOLLOWING EXOCYTOSIS}

As shown in Figure 4, neurons fixed during $20 \mathrm{~Hz}$ stimulation had some dense core vesicles with omega-shaped structures, suggesting exo/endocytosis. In addition, adjacent to the release sites we found clear vesicles and cisternae, thus suggesting that these structures were formed also as result of endocytosis. To explore if dense core vesicles also underwent endocytosis, the extracellular fluid was added with peroxidase before stimulation and peroxidase was detected by colloidal gold-coupled antibodies. As shown in Figure 5, multiple dense core vesicles became labeled, confirming endocytosis of full dense core vesicles and also supporting that large numbers of vesicles in the cluster become fused upon electrical stimulation.

The relative restriction of colloidal gold electron dense spheres to dense core vesicles and multivesicular bodies (Figures 5A,B) indicates that the staining was highly specific. In addition we also detected some gold labeling in the membrane of endoplasmic reticulum but not free within the cytoplasm (Figure 5A). That gold label was also present inside multivesicular bodies (Figures 5C,D) suggests that they contained vesicles that had undergone exo-and endocytosis, and that vesicle degradation upon endocytosis plays a major role in dense core vesicle cycling associated to somatic serotonin secretion by Retzius neurons.

\section{FORMATION OF MULTIVESICULAR BODIES}

The formation of multivesicular bodies has been studied in a variety of cell types (for review, see Piper and Katzmann, 2007). Our electron micrographs fit with the general scheme by indicating that multivesicular bodies form when membranous structures, including vesicles, are surrounded by microtubules and endoplasmic reticulum. In Figure 1 it was shown that after $20 \mathrm{~Hz}$ stimulation multivesicular bodies appear within the vesicle clusters near the plasma membrane. Figure 6 shows several examples of multivesicular bodies in the periphery of vesicle clusters from neurons fixed $10 \mathrm{~min}$ after electrical stimulation at $20 \mathrm{~Hz}$. Individual or aligned dense core vesicles are associated to multivesicular bodies (see also Figure 1), at parts in which they still seem to be opened. Some multivesicular bodies contain dense core and clear vesicles (Figure 6A). As shown above (Figure 5), multivesicular bodies formed by neurons stimulated in the presence of external 

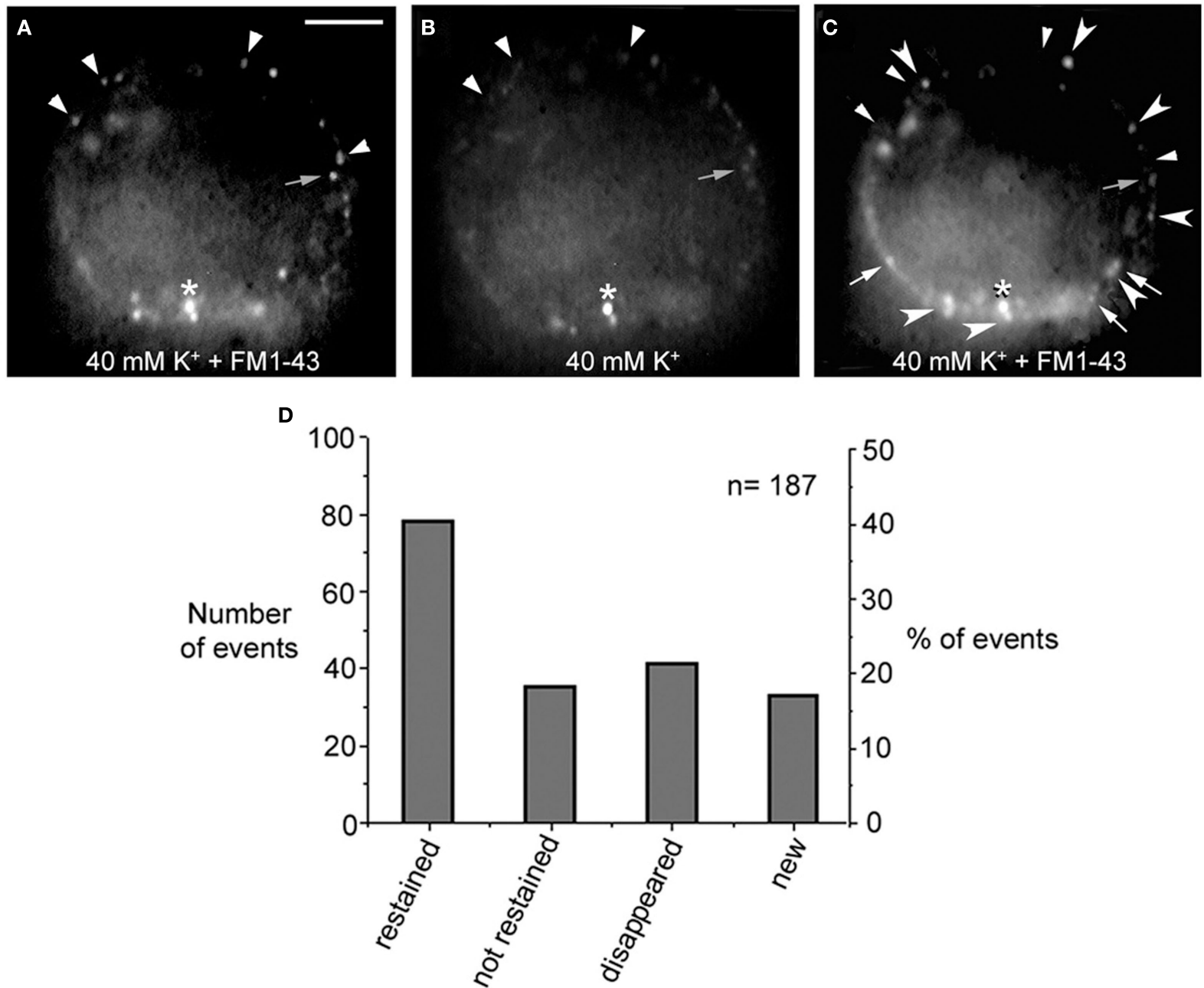

FIGURE 3 | FM1-43 staining upon subsequent depolarizations.

(A) Equatorial image of the soma of a cultured neuron that had been depolarized with $40 \mathrm{mM}$ potassium in the presence of FM1-43 in the external solution. Ten minutes after depolarization the dye was washed out from the fluid and neurons displayed their characteristic spotted pattern. Labeled spots are marked with the small arrowheads and small gray arrow. The asterisk marks an unspecific spot that was used as a reference for the following images in the series. (B) The FM1-43 fluorescence was partially photobleached to allow the testing of whether spots that were originally stained became restained. (C) After a second depolarization in the presence of fresh external FM1-43, some of the spots were restained (large arrowheads), some were not restained but could still be detected in their previous position (gray small arrows), others disappeared completely (small arrowheads), and others were newly formed (white arrows). Scale bar $=20 \mu \mathrm{m}$. (D) Graph of the distribution of the total number and percentage of fluorescent spots in each category described above. peroxidase and their surrounding vesicles also displayed electron dense gold particles. This also occurred in multivesicular bodies in the apparent process of formation shown in Figures 6C,D and is consistent with the early observations that multivesicular bodies are capable to accumulate peroxidase (Rosenbluth and Wissig, 1964; Holtzman and Peterson, 1969; Birks et al., 1972; Weldon, 1975).

\section{INTRACELLULAR MOBILIZATION OF FM1-43 FLUORESCENCE AFTER STIMULATION}

The destiny of the material contained inside multivesicular bodies remains a matter of debate (for review, see Von Bartheld and Altick, 2011), however, that multivesicular bodies in our electromicrographs appear in the perinuclear region suggest they have been transported after their peripheral formation. To explore if after electrical stimulation vesicles and or multivesicular bodies become transported toward the plasma region, FM1-43 fluorescent spots of four neurons that had been stimulated at $20 \mathrm{~Hz}$ were imaged in the same focal planes 12,36 , and $48 \mathrm{~h}$ after stimulation (Figure 7). At the end of the experiments the neurons were known to be healthy because they had good resting potentials and the capacity to produce normal action potentials. As shown in Figure 7, at all times tested, fluorescence remained restricted to small spots, and the label gradually appeared in more central parts of the soma, while there was a decrease in the number of peripheral spots. The transport pathway (as exemplified by the sequence of images made at a depth of $20 \mu \mathrm{m}$ from the plane of contact of the cell with the culture dish), produced a large concentration of 


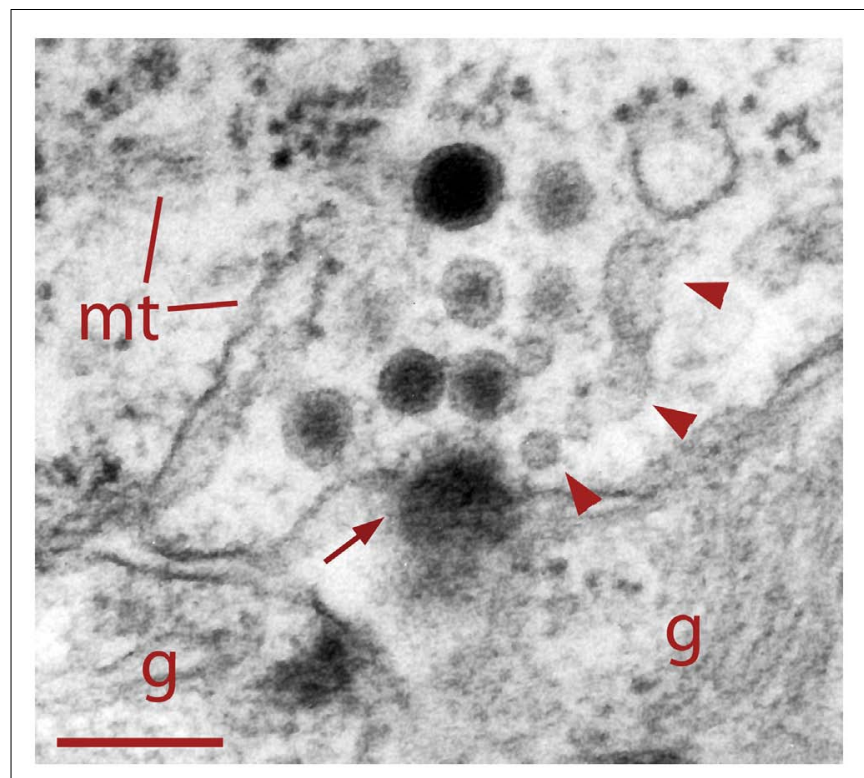

FIGURE 4 | Dense core vesicle fusion after electrical stimulation (arrow). To the right of the fusing vesicle are small clear vesicles and cisternae (arrowheads) atypical of somatic clusters before electrical stimulation. This suggests vesicle formation as part of the endocytic pathway. Glial cell fingers (g) can be seen outside the Retzius cell. Scale bar $=250 \mathrm{~nm}$.

spots at the center of the soma. This suggests that the mobilization of the spots did not occur randomly but followed well-organized patterns.

\section{ASSOCIATIONS BETWEEN MULTIVESICULAR BODIES, THE GOLGI APPARATUS, AND THE NUCLEUS}

Correlating with the previous result, in neurons fixed $10 \mathrm{~min}$ after electrical stimulation had ended, multivesicular bodies were abundant deep in the perinuclear region, near Golgi apparatuses, vesicles, and mitochondria (Figure 8, see also Figure 1), right in the regions in which vesicles seem to be forming, as described above. The multivesicular bodies in some of the sections established links with Golgi elements, endoplasmic reticulum, and mitochondria. Their vesicle contents and degradation products with irregular shapes were apparently extruded from the multivesicular bodies and became a continuous with the vesicle accumulations aligned with the Golgi apparatus (Figure 8A). Multivesicular body fragments also appeared in the perinuclear region, making contacts with the nuclear membrane (Figure 8B). These structural associations suggest that the multivesicular body contents were recycled in the Golgi apparatus and also that some of their content contributed to communication between the nucleus and the extracellular space.

\section{DISCUSSION}

We studied the cycling of dense core vesicles associated with serotonin release through exocytosis by serotonergic leech neurons. Dense core vesicles are distributed in a perinuclear pool where they seem to be formed and also in a pool of peripheral clusters. Electrical stimulation induces the transport of dense core vesicle clusters toward the plasma membrane, with which vesicles fuse. Endocytosis generates dense core and clear vesicles, which are later incorporated into multivesicular bodies. In turn, multivesicular bodies are transported back to the perinuclear region, where their contents seem to be recycled by the Golgi apparatus.

Somatic exocytosis of serotonin is a highly regulated process in neurons of vertebrates and invertebrates, in which serotonincontaining vesicles are mobilized centrifugally in response to electrical stimulation and then fused (De-Miguel and Trueta, 2005; Kaushalya et al., 2008; see review by Trueta and De-Miguel in this issue). In Retzius neurons, stimulation with series of $20 \mathrm{~Hz}$ trains at $2 \mathrm{~min}$ intervals produces two sigmoidal exocytosis kinetics with a duration of hundreds of seconds, with each sigmoidal expressing the arrival of one vesicle cluster (P. Noguez, C. Bustos, and F. F. De Miguel, in preparation).

These kinetics seem to reflect the subsequent arrival of two vesicle clusters at the same release site, upon their mobilization and fusion in response to electrical stimulation. This also provides a good explanation for the second staining experiment (Figure 3), in which clusters of vesicles stained with FM1-43 and then bleached became restained again upon a second depolarization in the presence of the fluorescent dye. Since the vesicle pool surrounding the nucleus remains after electrical stimulation, it is possible that the clusters arriving at the plasma membrane in response to electrical stimulation belong to the peripheral pool. However, that some of the fluorescent FM1-43 spots remain peripheral after so many hours following electrical stimulation also suggest that some multivesicular bodies are not transported retrogradely or that not all the dense core vesicles are incorporated into multivesicular bodies after exocytosis. Therefore, some may be degraded locally or free vesicles remain and are reused later, while multivesicular bodies traveling back to the perinuclear region are destroyed there or their material is reused for new vesicle synthesis.

Since about 100 vesicle clusters become fused upon a single $20 \mathrm{~Hz}$ train (Trueta et al., 2003) and each cluster contains 1001000 vesicles (P. Noguez, I. Santamaría-Holek, M. Rubí, and F. F. De Miguel, in preparation), we estimate that tens of thousands of vesicles fuse in response to a $20-\mathrm{Hz}$ train, with each vesicle containing 67,000 molecules of serotonin (Bruns and Jahn, 1995). This adds to the serotonin secreted from other extrasynaptic sites including the axons and dendrite varicosities. An equivalent amount of serotonin is secreted by the other Retzius neuron in the ganglion, since both respond in parallel upon the activation of common synaptic inputs (Velázquez-Ulloa et al., 2003). Therefore it is not surprising that a neuron pair is capable of producing the multiple modulatory effects of serotonin seen in the leech (Willard, 1981; Kristan and Nusbaum, 1982; Burrell et al., 2002; Crisp and Mesce, 2006; Calviño and Szczupak, 2008; Bisson and Torre, 2011).

Serotonergic neurons fail to fire at high frequencies and have developed mechanisms of autoinhibition that are triggered by serotonin release (Aghajanian and Lakoski, 1984; Heinrich et al., 1999; Rose et al., 2006; Gocht and Heinrich, 2007; Cercós et al., 2009). This, in addition to the depletion of releasable vesicle pools, shows that serotonin exocytosis from extrasynaptic sites operates with a high degree of autoregulatory mechanisms. In this regard, that dense core vesicles do not re-enter immediately the 


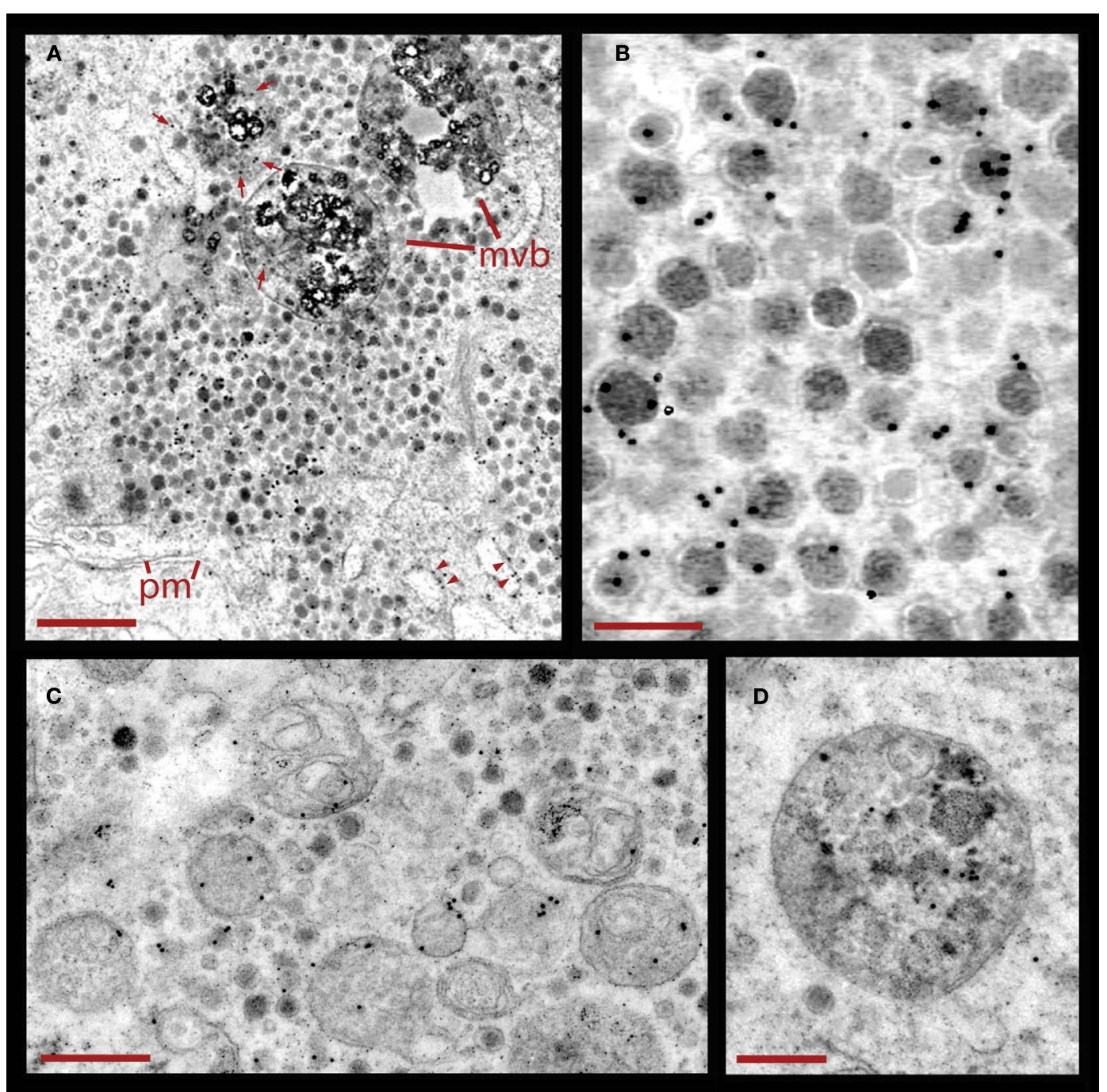

\section{FIGURE 5 | Endocytosis of peroxidase following electrical}

stimulation. (A) Vesicle cluster in a neuron fixed during stimulation in the presence of external peroxidase. Large multivesicular bodies (mvb) are also present. Peroxidase was detected by a polyclonal antibody coupled to $10 \mathrm{~nm}$ gold particles. The black electron dense spots are associated with vesicles in the clusters located near the plasma membrane (pm), and labeling was considerably lower outside of the cluster. However, multivesicular bodies (arrows) and membranes of endoplasmic reticulum (arrowheads) also displayed gold particles. Scale $=1.0 \mu \mathrm{m}$. (B) Higher magnification of a vesicle cluster with electron dense spots accumulated over the vesicles. Scale $=200 \mathrm{~nm}$. (C) Cluster with dense core vesicles and multivesicular bodies bearing gold particles. Scale $=1 \mu \mathrm{m}$. (D) Higher magnification of a multivesicular body that accumulated gold particles. Scale $=500 \mathrm{~nm}$ releasable pool but are packed, degraded, and transported towards the nucleus, contributes to this scheme by increasing the latency of vesicle replenish upon subsequent trains of electrical activity, when compared to the fast recycling occurring in synaptic vesicles (Betz and Bewick, 1992, 1993; Betz et al., 1992; Cochilla et al., 1999; Richards et al., 2000).

The clustering of dense core vesicle offers several advantages over having the same number of individual vesicles scattered, since their transport becomes more efficient and the plasma membrane specializations for vesicle fusion are concentrated at specific locations instead of being randomly distributed, thus increasing the efficiency of vesicle docking. Moreover, a localized release renders higher serotonin concentrations at particular release sites. Since serotonin is released onto surrounding glia, it may be proposed that glial cells regulate the diffusion of serotonin released from the soma, at least to the rest of the neurons wrapped within the same glial processes. This may also contribute to enlarge the timing of the serotonin effects on the central nervous system. Extrasynaptic communication or volume transmission between neurons and glia have been demonstrated already for different transmitter systems (Bjelke et al., 1996; Agnati et al., 2007, 2010; Pérez de la Mora et al., 2008; Araque and Navarrete, 2010; Fuxe et al., 2010, 2012).

The distance that vesicle clusters must move to reach the plasma membrane adds a lag to exocytosis, desynchronizes it from electrical activity and makes it slow compared to that from serotonergic 


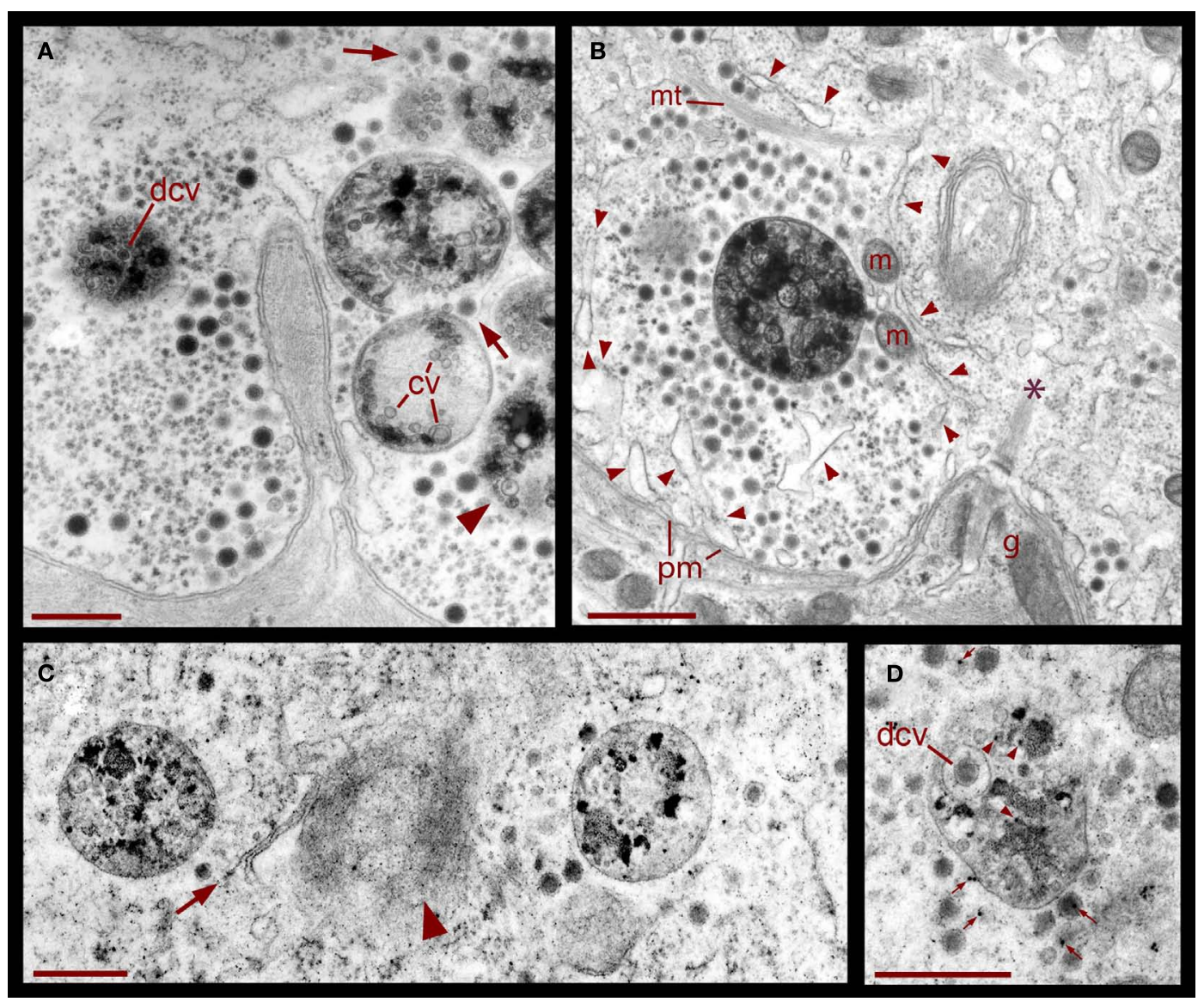

\section{FIGURE 6 | Formation of multivesicular bodies after electrical} stimulation. (A) Several multivesicular bodies near the plasma membrane with dense core vesicles around. The neuron was stimulated at $20 \mathrm{~Hz}$ and fixed 10 min later. Arrows point to vesicles associated with different multivesicular bodies, as they seem to be forming through the wrapping of endoplasmic reticulum. The arrowhead points to a multivesicular body containing different types of vesicles without a noticeable external layer of membranes. Clear vesicles (cv) and dense core vesicles (dcv) inside the multivesicular bodies can also be seen. (B) Multivesicular body surrounded by a radial array of dense core vesicles, endoplasmic reticulum (arrowheads), microtubules (mt), and mitochondria (m). Asterisk marks a bundle of microtubules attached to the plasma membrane through a hemi-desmosome. Stimulation was as in (A). (C) Formation of a multivesicular body (arrow) with microtubule coiling (arrowhead). (D) A multivesicular body after the neuron was stimulated in the presence of extracellular peroxidase, displaying gold marks, and containing clear and dense core vesicles (dcv). Arrowheads point to gold particles inside multivesicular bodies and small arrows point to gold particles on top of vesicles and membrane corpses, maybe produced upon endocytosis. Scale bar $=1 \mu \mathrm{m}$. synapses, in which events occur within the characteristic millisecond time scale (Fuchs et al., 1982; Henderson et al., 1983; Dietzel et al., 1986). This distance may determine at least in part the slow time course of secretion, which is similar to that of somatic exocytosis in other types of neurons (Zhang et al., 2007; Kaushalya et al., 2008; Colgan et al., 2009; Patel et al., 2009), and also by excitable endocrine cells (Penner and Neher, 1988; Heinemann et al., 1993; Chow et al., 1996; Rosé et al., 2002). This apparent type of microtubule-coupled transport suggests its dependence on kinesin motors. Therefore, somatic secretion has high energy requirements. On the way back to the perinuclear region, multivesicular bodies may also use microtubule backward transport, in this case coupled to dynein motors (van den Berg and Hoogenraad, 2012), thus increasing the energy expenses of exocytosis. That multivesicular bodies release their contents to the cytoplasm has been a provoking hypothesis since early studies (Palay, 1960).
Our data are in agreement with this possibility and also suggest that multivesicular bodies in perinuclear regions may release their contents, which in turn are used for new vesicle synthesis and also for providing the nucleus with information from the extracellular space. However, we do not have any evidence that multivesicular bodies may serve as releasible pools, as has been proposed for other cell types (for review, see Von Bartheld and Altick, 2011; Fuxe et al., 2012), since we have never seen multivesicular bound to the plasma membrane after electrical stimulation and on the contrary, multivesicular bodies seem to be formed as result of vesicle endocytosis and moving toward the perinuclear regions of the neuron.

The formation of new vesicles in the perinuclear region also contributes to the regulated secretion of serotonin by imposing the large migratory distances of the vesicle clusters to reach their fusion sites. One of the several questions yet to be answered is 

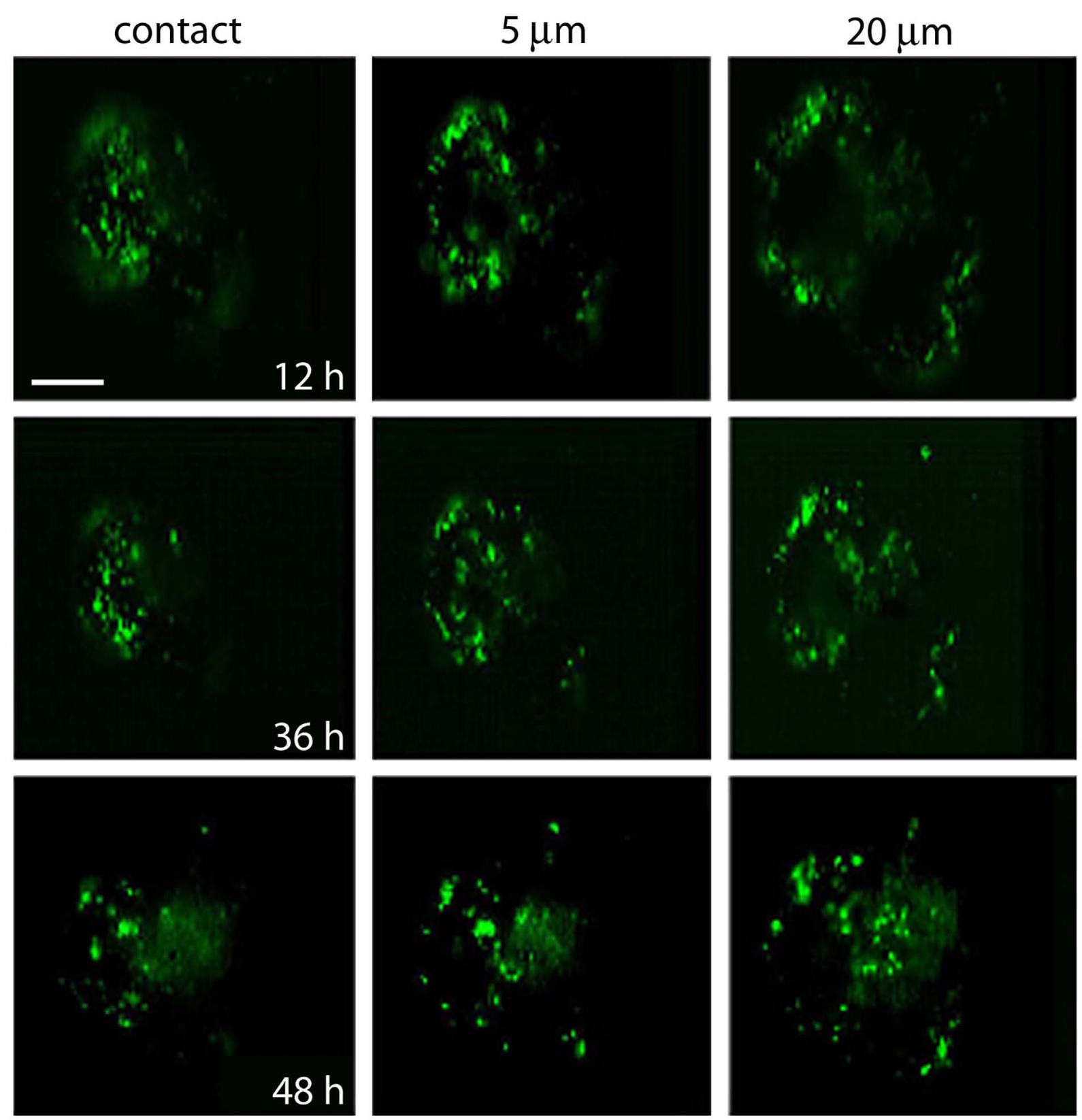

FIGURE 7 | Migration of fluorescent FM1-43 spots. Images taken from the site of contact with the dish bottom (left) and at 5 and $20 \mu \mathrm{m}$ depth. Each area was imaged at 12,36 , and $48 \mathrm{~h}$ after depolarization of cultured neurons in bathing solution containing $40 \mathrm{mM}$ potassium and FM1-43. Note the accumulation of spots to more internal regions of the soma at the $20-\mu \mathrm{m}$ focal plane. Scale bar $=20 \mu \mathrm{m}$. whether electrical activity induces the formation of new vesicles. This is a feasible possibility since electrical stimulation at high frequency evokes calcium-induced calcium release (Trueta et al., 2004) and calcium waves that reach the nucleus of Retzius neurons (Beck et al., 2001; C. Leon and F. F. De-Miguel, in preparation). Calcium in the nucleus activates gene expression (Bito et al., 1997; Wiegert and Bading, 2011), and therefore its role in vesicle cycling may include the activation of the vesicle formation, followed by their transport, exo- and endocytosis. Additional signals activating new vesicle synthesis may arrive with multivesicular bodies after vesicle endocytosis.

\section{GENERAL SIGNIFICANCE}

Somatic exocytosis is now recognized as a more general form of neuronal communication of central and peripheral neurons of vertebrates and invertebrates (see review by Trueta and De-Miguel in this issue). Since neuronal somatic secretion of transmitters and peptides occurs from dense core vesicles in different cells types (Huang and Neher, 1996; Zaidi and Matthews, 1997, 1999; Puopolo et al., 2001; Huang et al., 2007), the data presented here provides an example as to how the complexity of dense core vesicle cycling contributes to the regulation a fundamental mode of neuronal exocytosis and communication. 


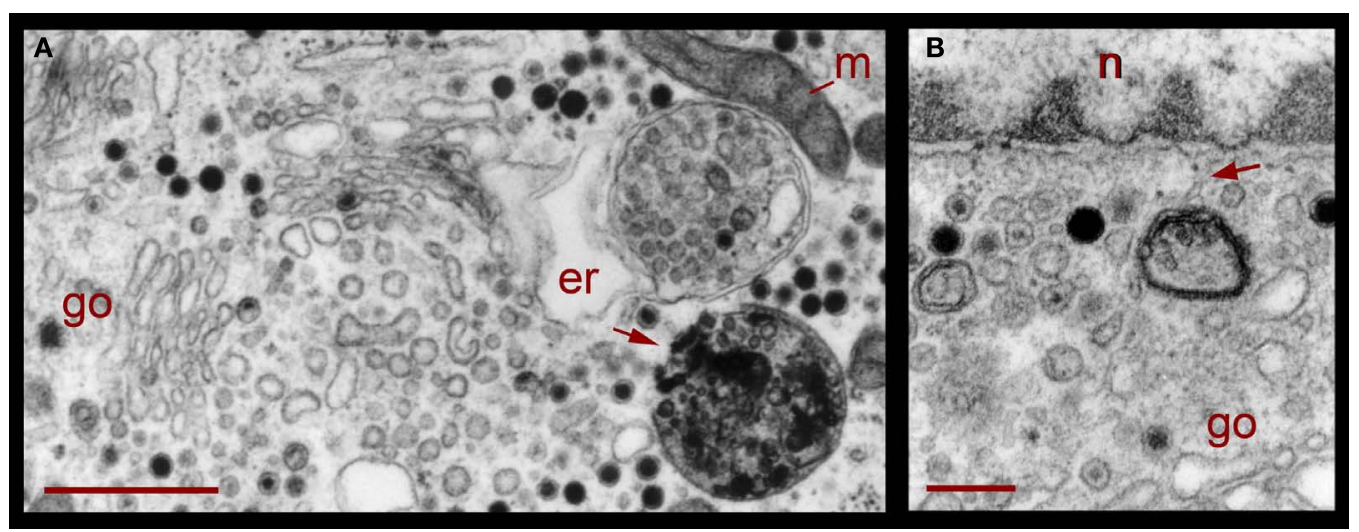

FIGURE 8 | Perinuclear multivesicular bodies. (A) Multivesicular bodies (mvb) coexisting with Golgi apparatus (go). The multivesicular body on the right top contains mostly clear vesicles and is in association with endoplasmic reticulum (er) and mitochondria ( $\mathrm{m}$ ). The one in the right bottom has a membrane discontinuity (arrowhead). On the left side of the image, small vesicles near the Golgi endings (asterisks) display electrodense material inside. (B) Contents of the multivesicular bodies are in association with the nuclear membrane and near Golgi apparatus ( $n=$ nucleus). The arrowhead points to structures possibly binding the multivesicular body fragment with the nuclear membrane. Scale bars $=500 \mathrm{~nm}$.

\section{ACKNOWLEDGMENTS}

We wish to express our gratitude to Mr. Bruno Mendez for his invaluable technical assistance during the whole project and to the electron microscopy and computer units of IFC-UNAM, in particular to Rodolfo Paredes and Francisco Perez, for their outstanding technical support to this project. This work was founded by DGAPA-UNAM grant IN211511 and CONACYT GRANT 130031 to Francisco F. De-Miguel.

\section{REFERENCES}

Aghajanian, G. K., and Lakoski, J. M. (1984). Hyperpolarization of serotonergic neurons by serotonin and LSD: studies in brain slices showing increased $\mathrm{K}+$ conductance. Brain Res. 305, 181-185.

Agnati, L. F., Genedani, S., Leo, G., Forni, A., Woods, A. S., Filaferro, M., Franco, R., and Fuxe, K. (2007). Abeta peptides as one of the crucial volume transmission signals in the trophic units and their interactions with homocysteine. Physiological implications and relevance for Alzheimer's disease. J. Neural Transm. 114, 21-31.

Agnati, L. F., Guidolin, D., Guescini, M., Genedani, S., and Fuxe, K. (2010). Understanding wiring and volume transmission. Brain Res. Rev. 64, 37-59.

Agnati, L. F., Leo, G., Zanardi, A., Genedani, S., Rivera, A., Fuxe, K., and Guidolin, D. (2006). Volume transmission and wiring transmission from cellular to molecular networks: history and perspectives. Acta Physiol. (Oxf.) 187, 329-344.

Araque, A., and Navarrete, M. (2010). Glial cells in neuronal network function. Philos. Trans. R. Soc. Lond. B Biol. Sci. 365, 2375-2381.

Beck, A., Lohr, C., and Deitmer, J. W. (2001). Calcium transients in subcompartments of the leech Retzius neuron as induced by single action potentials. J. Neurobiol. 48, 1-18.

Betz, W. J., and Bewick, G. S. (1992). Optical analysis of synaptic vesicle recycling at the frog neuromuscular junction. Science 255, 200-203.

Betz, W. J., and Bewick, G. S. (1993). Optical monitoring of transmitter release and synaptic vesicle recycling at the frog neuromuscular junction. J. Physiol. 460, 287-309.

Betz, W. J., Bewick, G. S., and Ridge, R. M. (1992). Intracellular movements of fluorescently labeled synaptic vesicles in frog motor nerve terminals during nerve stimulation. $\mathrm{Neu}$ ron $9,805-813$.

Birks, R. I., MacKey, M. C., and Weldon, P. R. (1972). Organelle formation from pinocytotic elements in neurites of cultured sympathetic ganglia. J. Neurocytol. 1, 311-340.

Bisson, G., and Torre, V. (2011). Statistical characterization of social interactions and collective behavior in medicinal leeches. J. Neurophysiol. 106, 78-90.

Bito, H., Deisseroth, K., and Tsien, R. W. (1997). Ca2 ${ }^{+}$-dependent regulation in neuronal gene expression. Curr. Opin. Neurobiol. 7, 419-429.

Bjelke, B., Goldstein, M., Tinner, B., Andersson, C., Sesack, S. R., Steinbusch, H. W., Lew, J. Y., He, X., Watson, S., Tengroth, B., and Fuxe, K. (1996). Dopaminergic transmission in the rat retina: evidence for volume transmission. J. Chem. Neuroanat. 12, 37-50.

Bruns, D., and Jahn, R. (1995). Realtime measurement of transmitter release from single synaptic vesicles. Nature 377, 62-65.

Burrell, B. D., Sahley, C. L., and Muller, K. J. (2002). Differential effects of serotonin enhance activity of an electrically coupled neural network. J. Neurophysiol. 87, 2889-2895.

Calviño, M. A., and Szczupak, L. (2008). Spatial-specific action of serotonin within the leech midbody ganglion. J. Comp. Physiol. A Neuroethol. Sens. Neural Behav. Physiol. 194, 523-531.

Cercós, M. G., De-Miguel, F. F., and Trueta, C. (2009). Real-time measurements of synaptic autoinhibition produced by serotonin release in cultured leech neurons. J. Neurophysiol. 102, 1075-1085.

Ceridono, M., Ory, S., Momboisse, F., Chasserot-Golaz, S., Houy, S., Calco, V., Haeberlé, A.-M., Demais, V. Bailly, Y., Bader, M. F., and Gasman, S. (2011). Selective recapture of secretory granule components after full collapse exocytosis in neuroendocrine chromaffin cells. Traffic 12 , 72-88.

Chazal, G. and Ralston, H. J. III. (1987). Serotonin-containing structures in the nucleus raphe dorsalis of the cat: an ultrastructural analysis of dendrites, presynaptic dendrites, and axon terminals. J. Comp. Neurol. 259, 317-329.

Chow, R. H., Klingauf, J., Heinemann, C., Zucker, R. S., and Neher, E. (1996). Mechanisms determining the time course of secretion in neuroendocrine cells. Neuron 16 , 369-376.

Cochilla, A. J., Angleson, J. K., and Betz, W. J. (1999). Monitoring secretory membrane with FM1-43 fluorescence. Annu. Rev. Neurosci. 22, $1-10$.

Coggeshall, R. E. (1972). Autoradiographic and chemical localization of 5-hydroxytryptamine in identified neurons in the leech. Anat. Rec. 172, 489-498.

Coggeshall, R. E., and Fawcett, D. W. (1964). The fine structure of the central nervous system of the leech, Hirodu medicinalis. J. Neurophysiol. 27, 229-289.

Coggeshall, R. E., and Lekan, H. A. (1996). Methods for determining numbers of cells and synapses: a case for more uniform standards of review. J. Comp. Neurol. 364, 6-15.

Colgan, L. A., Putzier, I., and Levitan, E. S. (2009). Activity-dependent vesicular monoamine transportermediated depletion of the nucleus supports somatic release by serotonin neurons. J. Neurosci. 29, 15878-15887. 
Crisp, K. M., and Mesce, K. A. (2006). Beyond the central pattern generator: amine modulation of decisionmaking neural pathways descending from the brain of the medicinal leech. J. Exp. Biol. 209(Pt 9), 1746-1756.

De-Miguel, F. F., and Trueta, C. (2005). Synaptic and extrasynaptic secretion of serotonin. Cell. Mol. Neurobiol.25, 297-312.

Descarries, L., and Mechawar, N. (2000). Ultrastructural evidence for diffuse transmission by monoamine and acetylcholine neurons of the central nervous system. Prog. Brain Res. 125, 27-47.

Dietzel, I. D., Drapeau, P., and Nicholls, J. G. (1986). Voltage dependence of 5-hydroxytryptamine release at a synapse between identified leech neurones in culture. J. Physiol. 372, 191-205.

Fernández-de-Miguel, F., and Drapeau, P. (1995). Synapse formation and function: insights from identified leech neurons in culture. J. Neurobiol. 27, 367-379.

Fuchs, P. A., Henderson, L. P., and Nicholls, J. G. (1982). Chemical transmission between individual Retzius and sensory neurones of the leech in culture. J. Physiol. 323, 195-210.

Fuxe, K., and Agnati, L. F. (eds). (1991). Volume Transmission in the Brain: Novel Mechanisms of Neuronal Transmission. New York: Raven Press.

Fuxe, K., Borroto-Escuela, D. O., Romero-Fernandez, W., Ciruela, F., Manger, P., Leo, G., Díaz-Cabiale, Z., and Agnati, L. F. (2012). On the Role of Volume Transmission and Receptor-Receptor Interactions in Social Behaviour: Focus on Central Catecholamine and Oxytocin Neurons. Brain Research. Available at: http://www.ncbi.nlm.nih.gov/ pubmed/22373652 [accessed April 12, 2012].

Fuxe, K., Dahlström, A. B., Jonsson, G., Marcellino, D., Guescini, M., Dam, M., Manger, P., and Agnati, L. (2010). The discovery of central monoamine neurons gave volume transmission to the wired brain. Prog. Neurobiol. 90, 82-100.

Gocht, D., and Heinrich, R. (2007). Postactivation inhibition of spontaneously active neurosecretory neurons in the medicinal leech. J. Comp. Physiol. A Neuroethol. Sens. Neural Behav. Physiol. 193, 347-361.

Heinemann, C., Rüden, L., Chow, R. H., and Neher, E. (1993). A twostep model of secretion control in neuroendocrine cells. Pflugers Archiv. 424, 105-112.

Heinrich, R., Cromarty, S. I., Hörner, M., Edwards, D. H., and Kravitz, E. A. (1999). Autoinhibition of serotonin cells: an intrinsic regulatory mechanism sensitive to the pattern of usage of the cells. Proc. Natl. Acad. Sci. U.S.A. 96, 2473-2478.

Henderson, L. P. (1983). The role of 5-hydroxytryptamine as a transmitter between identified leech neurones in culture. J. Physiol. 339, 309-324.

Henderson, L. P., Kuffler, D. P., Nicholls, J., and Zhang, R. (1983). Structural and functional analysis of synaptic transmission between identified leech neurones in culture. J. Physiol. 340, 347-358.

Herzog, V., and Farquhar, M. G. (1977). Luminal membrane retrieved after exocytosis reaches most golgi cisternae in secretory cells. Proc. Natl. Acad. Sci. U.S.A. 74, 5073-5077.

Herzog, V., and Miller, F. (1979). Membrane retrieval in epithelial cells of isolated thyroid follicles. Eur. J. Cell Biol. 19, 203-215.

Holtzman, E., and Peterson, E. R. (1969). Uptake of protein by mammalian neurons. J. Cell Biol. 40, 863-869.

Huang, H.-P., Wang, S.-R., Yao, W., Zhang, C., Zhou, Y., Chen, X.-W., Zhang, B., Xiong, W., Wang, L. Y., Zheng, L. H., Landry, M., Hökfelt, T., Xu, Z. Q., and Zhou, Z. (2007). Long latency of evoked quantal transmitter release from somata of locus coeruleus neurons in rat pontine slices. Proc. Natl. Acad. Sci. U.S.A. 104, 1401-1406.

Huang, L. Y., and Neher, E. (1996). $\mathrm{Ca}(2+)$-dependent exocytosis in the somata of dorsal root ganglion neurons. Neuron 17, 135-145.

Iversen, L. L. (1971). Role of transmitter uptake mechanisms in synaptic neurotransmission. Br. J. Pharmacol. 41, 571-591.

Kaushalya, S. K., Desai, R., Arumugam, S., Ghosh, H., Balaji, J., and Maiti, S. (2008). Three-photon microscopy shows that somatic release can be a quantitatively significant component of serotonergic neurotransmission in the mammalian brain. J. Neurosci. Res. 86, 3469-3480.

Kristan, W. B. Jr., and Nusbaum, M. P. (1982). The dual role of serotonin in leech swimming. J. Physiol. (Paris) 78, 743-747.

Kuffler, D. P., Nicholls, J., and Drapeau, P. (1987). Transmitter localization and vesicle turnover at a serotoninergic synapse between identified leech neurons in culture. J. Comp. Neurol. 256, 516-526.

Nicholls, J. G., and Kuffler, D. P. (1990). Quantal release of serotonin from presynaptic nerve terminals. Neurochem. Int. 17, 157-163.

Palay, S. L. (1960). The fine structure of secretory neurons in the preoptic nucleus of the goldish (Carassius auratus). Anat. Rec. 138 417-443.

Park, J. J., Koshimizu, H., and Loh, Y. P. (2009). Biogenesis and transport of secretory granules to release site in neuroendocrine cells. J. Mol. Neurosci. 37, 151-159.

Patel, J. C., Witkovsky, P., Avshalumov, M. V., and Rice, M. E. (2009). Mobilization of calcium from intracellular stores facilitates somatodendritic dopamine release. J. Neurosci. 29, 6568-6579.

Patzak, A., Aunis, D., and Langley, K. (1987). Membrane recycling after exocytosis: an ultrastructural study of cultured chromaffin cells. Exp. Cell Res. 171, 346-356.

Penner, R., and Neher, E. (1988). The role of calcium in stimulussecretion coupling in excitable and non-excitable cells. J. Exp. Biol. 139 329-345.

Pérez de la Mora, M., Jacobsen, K. X., Crespo-Ramírez, M., FloresGracia, C., and Fuxe, K. (2008). Wiring and volume transmission in rat amygdala. Implications for fear and anxiety. Neurochem. Res. 33, 1618-1633.

Piper, R. C., and Katzmann, D. J. (2007) Biogenesis and function of multivesicular bodies. Annu. Rev. Cell Dev. Biol. 23, 519-547.

Puopolo, M., Hochstetler, S. E., Gustincich, S., Wightman, R. M., and Raviola, E. (2001). Extrasynaptic release of dopamine in a retinal neuron: activity dependence and transmitter modulation. Neuron 30, 211-225.

Richards, D. A., Guatimosim, C., and Betz, W. J. (2000). Two endocytic recycling routes selectively fill two vesicle pools in frog motor nerve terminals. Neuron 27, 551-559.

Rosé, S. D., Lejen, T., Casaletti, L., Larson, R. E., Pene, T. D., and Trifaró, J.-M. (2002). Molecular motors involved in chromaffin cell secretion. Ann. N. Y. Acad. Sci. 971, 222-231.

Rose, T., Gras, H., and Hörner, M. (2006). Activity-dependent suppression of spontaneous spike generation in the Retzius neurons of the leech Hirudo medicinalis L. Invert. Neurosci. 6, 169-176.
Rosenbluth, J., and Wissig, S. L. (1964). The distribution of exogenous ferritin in toad spinal ganglia and the mechanism of its uptake by neurons. J. Cell Biol. 23, 307-325.

Trueta, C., Méndez, B., and De-Miguel, F. F. (2003). Somatic exocytosis of serotonin mediated by L-type calcium channels in cultured leech neurones. J. Physiol. 547(Pt 2), 405-416.

Trueta, C., Sánchez-Armass, S., Morales, M. A., and De-Miguel, F. F. (2004). Calcium-induced calcium release contributes to somatic secretion of serotonin in leech Retzius neurons. J. Neurobiol. 61, 309-316.

van den Berg, R., and Hoogenraad, C. C. (2012). Molecular motors in cargo trafficking and synapse assembly. Adv. Exp. Med. Biol. 970, 173-196.

Velázquez-Ulloa, N., Blackshaw, S. E., Szczupak, L., Trueta, C., García, E., and De-Miguel, F. F. (2003). Convergence of mechanosensory inputs onto neuromodulatory serotonergic neurons in the leech. J. Neurobiol. 54, 604-617.

Von Bartheld, C. S., and Altick, A. L. (2011). Multivesicular bodies in neurons: distribution, protein content, and trafficking functions. Prog. Neurobiol. 93, 313-340.

Weldon, P. R. (1975). Pinocytotic uptake and intracellular distribution of colloidal thorium dioxide by cultured sensory neurites. J. Neurocytol. 4, 341-356.

Wiegert, J. S., and Bading, H. (2011). Activity-dependent calcium signaling and ERK-MAP kinases in neurons: a link to structural plasticity of the nucleus and gene transcription regulation. Cell Calcium 49, 296-305.

Willard, A. L. (1981). Effects of serotonin on the generation of the motor program for swimming by the medicinal leech. J. Neurosci. 1, 936-944.

Zaidi, Z. F., and Matthews, M. R. (1997). Exocytotic release from neuronal cell bodies, dendrites and nerve terminals in sympathetic ganglia of the rat, and its differential regulation. Neuroscience 80, 861-891.

Zaidi, Z. F., and Matthews, M. R. (1999). Stimulant-induced exocytosis from neuronal somata, dendrites, and newly formed synaptic nerve terminals in chronically decentralized sympathetic ganglia of the rat. J. Comp. Neurol. 415, 121-143.

Zhang, X., Chen, Y., Wang, C., and Huang, L.-Y. M. (2007). Neuronal 
somatic ATP release triggers neuronsatellite glial cell communication in dorsal root ganglia. Proc. Natl. Acad. Sci. U.S.A. 104, 9864-9869.

Conflict of Interest Statement: The authors declare that the research was conducted in the absence of any commercial or financial relationships that could be construed as a potential conflict of interest.

Received: 13 April 2012; paper pending published: 26 April 2012; accepted: 14 May 2012; published online: 06 June 2012.
Citation: Trueta C, Kuffler DP and DeMiguel FF (2012) Cycling of dense core vesicles involved in somatic exocytosis of serotonin by leech neurons. Front. Physio. 3:175. doi: 10.3389/fphys.2012.00175

This article was submitted to Frontiers in Membrane Physiology and Biophysics, a specialty of Frontiers in Physiology.
Copyright (C) 2012 Trueta, Kuffler and De-Miguel. This is an open-access article distributed under the terms of the Creative Commons Attribution Non Commercial License, which permits noncommercial use, distribution, and reproduction in other forums, provided the original authors and source are credited. 